published as: Husmann, Julian, and Paul M. Näger. 2018. Physical Composition by Bonding. In $P e$ ter van Inwagen: Materialism, Free Will and God, ed. Ludger Jansen and Paul M. Näger, 65-96. Cham: Springer, DOI: 10.1007/978-3-319-70052-6_4.

\title{
Physical Composition by Bonding
}

\author{
Julian Husmann \\ Paul M. Näger \\ julian.husmann@wwu.de \\ paul.naeger@wwu.de \\ Department of Philosophy, WWU Münster, Germany
}

\begin{abstract}
Van Inwagen proposes that besides simples only living organisms exist as composite objects. This paper suggests expanding van Inwagen's ontology by also accepting composite objects in the case that physical bonding occurs (plus some extra conditions). Such objects are not living organisms but rather physical bodies. They include (approximately) the complete realm of inanimate ordinary objects, like rocks and tables, as well as inanimate scientific objects, like atoms and molecules, the latter filling the ontological gap between simples and organisms in van Inwagen's original picture. We thus propose a compositional pluralism claiming that composition arises if and only if bonding or life occurs.
\end{abstract}

Keywords Bonding, Composition, Mereology, Special composition question

\subsection{Introduction}

The world as we experience it seems to be one of parts and wholes. A tree has a trunk, branches and leaves as its parts, bricks arranged and cemented in an appropriate way make up a house, and scientists tell us that a knife consists of a large number of iron atoms bound together. While the concepts of "part" and "whole" belong to our standard repertoire of thinking, philosophers have contended that the relation between a whole and its parts, the relation of composition, is notoriously unclear. As with other puzzling relations like the causal relation, philosophers since antiquity have developed answers to the question of what the nature of this relation might be. Is it a fundamental one or can it be reduced to other facts? If the latter, what are the necessary and sufficient conditions of its obtaining? This main question of mereology is what Peter van Inwagen calls the general composition question. 
The game of this debate changed considerably when in 1987 van Inwagen first formulated the special composition question (SCQ; van Inwagen 1987). (As in many cases, philosophical progress here did not start with an answer, but rather with a new question.) Instead of enquiring what the nature of the composition relation is, he asked under what circumstances composition occurs; or put in a more practical way: What can one do to make some (non-overlapping) physical objects compose something? Think, for instance, about Lego bricks spread on a table, and suppose that we start arranging them in such a way that they form a (small) house. Is there any stage in this process when we can say that the bricks compose something? Answering this question does not require abstract metaphysical descriptions, but rather concrete conditions that make composition happen. This is one main reason why the SCQ-approach to the problem of composition has found many adherents; and it also explains why this approach has been so productive in the discussion about composition. ${ }^{1}$ This paper is about such answers to the SCQ.

Van Inwagen develops his own answer to the SCQ in his Material Beings (1990). He argues that only two kinds of things exist: fundamental material objects without parts (simples) and living organisms composed of these simples. So his explicit answer to the SCQ is that certain objects ("the $x \mathrm{~s}$ ") ${ }^{2}$ compose something "if and only if the activity of the $x$ s constitutes a life" (van Inwagen 1990, 82). This solution implies that inanimate natural composites like stones, planets and stars do not exist; and it also implies that (inanimate) artefacts like a Lego house, tables, cars and paintings do not exist either. According to van Inwagen, this is not to say, however, that in situations in which we normally would say that there is a stone or a table there is nothing at all; rather, in these cases, there are simples arranged stone-wise or table-wise, respectively. This also suggests a semantic reduction strategy for our usage of words like "stone", "table" etc.: Since tables do not exist, "table" cannot literally refer to something in the world; rather, all sentences about tables have to be translated into sentences about table-wise arranged simples. ${ }^{3}$

Van Inwagen considers his view a moderate answer to the SCQ because it avoids extreme positions: He neither accepts mereological universalism, which is to say that any $x \mathrm{~s}$

\footnotetext{
${ }^{1}$ Two recent examples discussing the SCQ are Thomasson (2007, esp. 126-136) and Carmichael (2015), who both defend a commonsense view, the latter by giving a series-style answer. The question has been productive even outside of metaphysics. Chant $(2006,422)$, for instance, applies the SCQ in action theory by asking under what conditions several actions compose an extensive action. This is what she calls "The Special Composition Question in Action".

${ }^{2}$ Van Inwagen (1990, 23-27) introduces "the $x \mathrm{~s}$ " as a plural name ("plural referring expression") to be able to refer to certain objects as a plurality without talking about contested entities like the set of these objects.

${ }^{3}$ But see Tallant (2014) for a critique of this strategy.
} 
under any conditions compose something (e.g., Lewis 1991), and especially he dismisses scattered objects; nor does he accept mereological nihilism, which is to hold that there are no composite objects whatsoever, i.e., there are no circumstances for any $x \mathrm{~s}$ under which the $x$ s compose something (e.g., Rosen and Dorr 2022 or Sider 2013). Rather, according to van Inwagen, there are $x$ s that compose something under specific conditions; and also, for any $x \mathrm{~s}$, there are conditions under which they do not compose anything. So any moderate answer to the SCQ must provide a criterion that separates cases of composition from cases in which composition does not occur. In this paper we shall presume that a moderate answer in this sense is the right way to answer the SCQ.

However, among conceivable moderate answers, van Inwagen's view might still be considered somewhat radical for being too close to nihilism: Of all the composite objects whose existence we usually assume, only organisms are said to exist - no rocks, no tables. And also, a majority of composite objects whose existence scientists typically assume - atoms, molecules, solid bodies, planets, stars - would not count as existent either. So an obvious question would be thus: Is it possible to find a trade-off between nihilism and universalism that is closer to a via media than van Inwagen suggests? More precisely, is it possible to formulate a criterion of composition according to which, besides living organisms, inanimate physical objects like atoms, rocks, stars and tables would exist as well? This will be the main question of this paper.

Searching for possible answers, we shall agree with van Inwagen in that plausible criteria of composition must obey the

Duplication principle: "If the $x$ s compose something, and if the $y$ s perfectly duplicate the $x \mathrm{~s}$ (both in their intrinsic properties and in the spatiotemporal and causal relations they bear to one another), then the $y$ s compose something." (van Inwagen 1990, 138)

The principle requires that whether certain objects ("the $x \mathrm{~s}$ ") compose something may only depend on their intrinsic properties and the relations among the $x \mathrm{~s} ;{ }^{4}$ emphatically, it may not depend in any way on properties of the environment or on relations of the $x \mathrm{~s}$ with the environment.

A second pillar for our investigation will be a scientific worldview. Thinking about which composite objects there are, we believe we should be informed about which composite objects scientists accept. Being able to reproduce those objects by our criterion of

\footnotetext{
${ }^{4} \mathrm{Cf}$. Lewis' definition of intrinsic properties: "If something has an intrinsic property, then so does any perfect duplicate of that thing; whereas duplicates situated in different surroundings will differ in their extrinsic properties." (Lewis 1983, 197) Van Inwagen reformulates this principle in the style of his SCQ to make it suitable for discussing composite objects.
} 
composition will be a benchmark for our theory. Another boundary constraint is to assume those objects as simples that current physics accepts as fundamental, i.e., the fundamental particles from the standard model (quarks and leptons) and their specific interactions (bosons). ${ }^{5}$ In this sense, our investigation here will be a study in empirically informed metaphysics (sometimes called "inductive metaphysics" or "naturalistic metaphysics").

Due to a lack of space, we shall not take into account the specific features of quantum theory. ${ }^{6}$ In particular, we shall neglect quantum entanglement, the identity of indiscernible objects and the spatial indefiniteness of quantum objects. The scientific picture we shall look at rather is the one that can be found in textbooks about (non-quantum) chemistry where the parts of atoms or molecules are treated as quasi-classical while all bonding effects, even if their nature is quantum mechanical (e.g., covalent bonds), are, of course, accounted for. So while neglecting certain quantum peculiarities, since bonding is a central concept in quantum theory as well, it is clear that the results of our study might be transferred to the quantum realm.

On the basis of the duplication principle and a scientific worldview we shall argue that one can expand van Inwagen's ontology of composite objects in an interesting and plausible way if one regards certain types of physical bonding as appropriate conditions under which composition occurs. (Note that we do not distinguish between physical and chemical bonding since the latter can be reduced to the former; so by "physical bonding" we mean all types of bonding due to natural forces.) This will yield atoms, molecules, solid bodies, tables, planets and galaxies as existing composite objects. However, we do not consider this new criterion as a competitor to van Inwagen's criterion of life. Rather than regarding bonding as a necessary and sufficient criterion for composition, we only consider it a sufficient one. If one relaxes van Inwagen's criterion to a sufficient (and not necessary) one as well, one can disjunctively combine the two: The $x$ s compose something if and only if either the $x$ s bind (plus some extra conditions) or the activity of the $x \mathrm{~s}$ constitutes a life. We shall discuss the prospects of such a compositional pluralism at the end of this paper.

\footnotetext{
${ }^{5}$ Note that our proposal does not depend on the fact that these currently accepted fundamental objects are indeed indivisible; it only depends on the fact that objects from possible deeper levels compose in a similar way to these "fundamental" particles, as these compose to higher objects. Presupposing that there is a fundamental level at which certain simples exist, we should say that we do not discuss the case that the world might be "gunky", i.e., infinitely divisible.

${ }^{6}$ For mereological questions in the quantum realm see, for instance, Maudlin (1998) or Calosi and Tarozzi (2014).
} 
Our argument proceeds in six steps. We first try to show that van Inwagen's arguments against certain types of bonding as composition criteria are inconclusive and leave room for alternative views (Sect. 4.2). Exploring these possibilities we first characterize bonding (and composition based on bonding) roughly as spatial confinement (Sect. 4.3). Investigating the scientific notion of bonding we show that bonding is more than just interaction (Sect. 4.5), namely a certain relation between kinetic and potential energy (Sect. 4.6). On the basis of this refined concept of bonding, we develop a suitable criterion of composition (Sect. 4.7). Finally, we spell out some consequences of our approach and discuss the results (Sect. 4.8).

\subsection{Van Inwagen's Dismissal of Bonding}

Before accepting life as a criterion of composition, van Inwagen (1990) discusses and discards several alternative criteria that might seem plausible:

(i) Contact (chap. 3)

(ii) Fastening (chap. 6)

(iii) Cohesion (chap. 6)

(iv) Fusion (chap. 6)

(v) Combinations of (ii)-(iv) ("series-style answers", chap. 7)

Each of these points represents a potential answer to the SCQ. Answer (i), for instance, suggests that some $x$ s compose something if and only if they are in contact. This criterion, however, as van Inwagen convincingly argues, has implausible consequences and should be discarded: Contact is a very unstable relation, and, especially from a scientific point of view, elementary particles neither have a shape nor, in general, a precise location; hence, it seems hopeless to apply the concept of contact to these objects (van Inwagen 1990, esp. 34-35). We agree with this evaluation; and we also agree with van Inwagen's arguments against series style answers (v) (which we shall not review since they are irrelevant for our argument in this paper). ${ }^{7}$ Hence, we concentrate on van Inwagen's discussion of (ii)(iv), which concern certain specific types of physical bonding.

\footnotetext{
${ }^{7}$ The idea of series-style proposals is to say that there are several types of relations $R_{1}, \ldots, R_{\mathrm{n}}$ (e.g., several types of bonding) as well as several types of objects $F_{1}, \ldots, F_{\mathrm{n}}$ that have to be discerned for answering the $\mathrm{SCQ}$; for the $x \mathrm{~S}$ of a certain kind $F_{\mathrm{i}}$ only compose something if they stand in the right kind of relation $R_{\mathrm{i}}$. More precisely:

$\exists y$ (the $x$ s compose $y$ ) if and only if the $x \mathrm{~s}$ are $F_{1}$ and stand in $R_{1}$, or the $x \mathrm{~s}$ are $F_{2}$ and stand in $R_{2}, \ldots$, or
} 
According to van Inwagen, two objects are fastened to each other (ii) when they "are in contact and [...] so arranged that, among all the many sequences in which forces of arbitrary directions and magnitudes might be applied to either or both of them, at most only a few would be capable of separating them without breaking or permanently deforming or otherwise damaging either of them" (van Inwagen 1990, 56).

Paradigms of fastened objects are those that are screwed, nailed, sewed or bolted to each other, for in all these cases only applying a force in a specific direction and strength to the screw, the nail, the fathom or the bolt will separate the two without destroying any of them. Cohering objects (iii), in contrast, are bound together but "can't be pulled apart, or even moved in relation to one another, without breaking some of them" (van Inwagen 1990, 58). Examples are two pieces of wood glued together or metal welded together. Fused objects (iv), ${ }^{8}$ finally, are "melt into each other in a way that leaves no discernable boundary" (van Inwagen 1990, 59). This might happen when "two very smooth pieces of chemically pure metal are brought together" (van Inwagen 1990, 59) and merge without any seam.

Van Inwagen adduces one main counterexample against bonding as a composition criterion, which he modifies according to the kind of bonding in question. He proposes considering situations with two persons who become physically bound to one another. Here is his example for the case of fastening:

Suppose again that you and I shake hands. [...] Now, suppose that the fingers of our hands were suddenly to become paralyzed, with the embarrassing consequence that we were unable to let go of each other. Suppose that, in fact, because of the paralysis of our entwined fingers, it had become impossible for anyone to pull us apart by main force, short of doing us damage. On any reasonable account, then, we have suddenly become fastened to each other. But it is certainly not true that an object composed of you and me comes into existence at the instant our fingers became paralyzed. Our paralysis has not added to the furniture of earth; it has merely diminished its capacity to be rearranged. Therefore, composition is not, primarily, a matter of things being fastened to one another. (van Inwagen 1990, 57-58)

The surprising fact about this passage is that van Inwagen provides the example and then just claims that in this case fastening does not lead to composition: He does not provide an explicit argument for his view, but rather seems to refer to intuitions. His description of the example in the case of cohesion is even scarcer:

the $x \mathrm{~s}$ are $F_{\mathrm{n}}$ and stand in $R_{\mathrm{n}}$.

Van Inwagen (1990, 64-66) presents convincing arguments against this kind of proposal.

${ }^{8}$ Unlike in other works on mereology, here fusion does not denote the mereological sum, but rather the very specific kind of bonding by merging. 
Suppose once more that you and I shake hands, this time after I have smeared my hand with one of those glues whose manufacturers warn us that they "bond skin instantly." No new thing comes to be in the course of our consequent painful adventure. (van Inwagen 1990, 58)

While the case of fusion is a bit more elaborated again, it still does not provide an explicit argument:

Consider Alice and Beatrice, who are identical twins. A mad surgeon cuts of Alice's left hand and Beatrice's right hand and joins their stumps together, so that they look rather as if they were part of a chain of paper dolls. The surgeon thus produces what might be described as a case of artificial Siamese twins. It is at least theoretically possible that the anatomy of Alice's wrist be so nearly an exact match to the anatomy of Beatrice's wrist, and the healing of one to the other be so nearly perfect, that no boundary between Alice and Beatrice be discoverable; it may be that there is a region such that there is simply no answer to the question whether the cells in that region are Alice's cells or Beatrice's cells. And yet, it seems to me, it is quite unreasonable to say that our mad surgeon has, like Dr. Frankenstein, created a new being by causing parts of existing beings to fuse. [...] Despite the fact that they are fused and separable only by surgery or sheer brutality, there is nothing but Alice and Beatrice[...]. (van Inwagen 1990, 59)

Van Inwagen summarizes his case against bonding relations:

Any bonding relation that can hold between any two moderate-sized specimens of dry goods can (I should think) hold between two human beings, and it is pretty clear that one cannot bring a composite object into existence by bonding two human beings - or two living organisms of any sort - to each other. (van Inwagen 1990, 62)

As witty and eloquent as these examples may be, van Inwagen clearly does not provide an argument on their basis. Why not? When discussing the case of contact, where he first introduces the situation of two persons shaking hands, he explains:

It is a basic conviction of mine that this theory [that two things compose a third at contact] is wrong and that its being wrong is in no sense a matter of convention. I cannot prove this thesis, for I know of no propositions more plausible than itself from which it could be derived. I can only say that I shall try to display in this book the fruits of agreeing with me about this and various similar theses. (van Inwagen 1990, 36)

So his reason for not presenting an argument against contact is that he does not have one; instead, the example with two persons shaking hands seems to be meant to push intuitions, ${ }^{9}$ and it seems obvious that in the case of adapting this counterexample to criteria with bonding he regards the situation in a fairly similar way.

\footnotetext{
${ }^{9}$ Van Inwagen's claim that he has no argument against contact might seem odd given that above we have presented his arguments from elementary particles lacking shape and definite location. The explanation seems to be that his argument from elementary particles presupposes quantum physics and after having presented that argument he asks to "imagine ourselves in a comfortable seventeenth-century physical world, a world
} 
The intuitions in question seem to be the following: When two persons' bodies become physically bound to one another, it is implausible to suggest that a third person, the sum of the two persons, comes into being. We agree with that. Nor is it plausible to hold that a super-organism is composed of the singular organisms. For in the cases of fastening and cohesion virtually all organic functions remain independent; and with exception of the circulatory system this is true even in the case of fusion. We also agree with this intuition.

We do not agree, however, with the conclusion that in such situations nothing is composed at all. Contrary to what van Inwagen suggests, we believe that bonding is an appropriate basis for composition and that in all three cases of bonding a new object does come into existence: If one abstracts from the fact that the joined objects are living bodies, they are first of all physical bodies in the rough sense of being a physical object whose parts are bound together (more on physical bodies in the following sections); and the thesis we shall argue for in this paper is that (non-overlapping) physical bodies $x \mathrm{~s}$ bound together do compose a whole, namely another physical body $y$, which has the $x \mathrm{~s}$ as parts. Following this line of thought will turn out to be intuitive - because it allows for the existence of usually accepted objects like rocks, tables and the moon - as well as to be in line with a scientific realist's view on what there is - claiming the existence of things like protons, atoms, molecules and stellar clouds. Assuming the existence of these objects also allows us to refer to these objects in quotidian statements or scientific theories such that extensive semantic reduction strategies become superfluous.

The position we shall suggest is not to discard van Inwagen's view altogether. We shall not say that some $x$ s whose activity constitutes a life do not compose something; life, in our view, still is a sufficient condition for composition. What we deny is that this condition is necessary, because bonding, in our view, is another sufficient condition. Formally, this will yield a disjunctive criterion of composition: The $x$ s compose something if and only if they either bind together (plus some extra conditions) or their activity constitutes a life. Materially, this amounts to accepting a kind of compositional pluralism: Besides simples, there are composite physical bodies, whose criterion of composition is based on bonding; and there are composite organisms, whose criterion of composition is based on life. We regard this position as an expansion of van Inwagen's view, which has the advantage of overcoming van Inwagen's closeness to mereological nihilism.

that consists entirely of physical objects of various sizes - solid objects having surfaces and made of stuffs" (van Inwagen 1990, 34). It is only in this restricted classical image that van Inwagen is left with his example of two people shaking hands and cannot deliver an argument against contact. The situation, however, is not similar in the cases of bonding: there he only presents the modified examples of shaking hands - so in those cases there really seems to be no argument at all. 


\subsection{A Scientific Ontology}

What kind of ontology are we looking for exactly? We have said that van Inwagen's view is close to nihilism because it only regards simples and organisms (including the cells of multicellular organisms) as existing. According to a standard contemporary scientific view, however, there are organs that are proper parts of complex organisms and have cells as their proper parts; (bio-)molecules are parts of cells; atoms are parts of (bio)molecules; protons, neutrons and electrons are parts of atoms; these electrons are simples according to the actual standard model of particle physics; protons and neutrons, however, have up- and down-quarks as parts, which, finally, are simples as well. In sum, what science suggests is that there are many more inanimate objects in between simples and organisms than van Inwagen accepts, and that these objects form a nested hierarchy of parts and wholes from quarks and electrons up to macroscopic organisms.

Moreover, according to this scientific ontology, besides composing living cells, molecules can also form inanimate solid objects like pieces of wood or plastic; and rather than composing molecules, certain kinds of atoms arrange in atomic lattices, which form grains of salt, crystals, rocks or pieces of metal. These are the kinds of things that our mid-sized inanimate ordinary objects are made of and which van Inwagen dismisses as well. And a huge amount of things of these kinds can compose a planet (or a star), a solar system, a galaxy - composites of this size do not occur in van Inwagen's picture either.

The question we shall pursue in the following is whether we can find a sufficient criterion of composition which accounts for the existence of all these inanimate composite objects that science (and common sense) assumes but van Inwagen misses. Our idea is that a suitable concept of bonding might account for them; for what all these composite objects seem to have in common is that their parts are somehow bound together: Nuclei bind to electrons to form atoms, atoms bind to form molecules, molecules bind to form cell membranes and so on.

\subsection{Bonding Implies Spatial Confinement}

What exactly is bonding? One of the main aims of this paper is to reconstruct a scientific view of bonding, and this will be the aim of the following Sect. (4.5, 4.6 and 4.7). Here we shall start with an intuitive operational characterization, which we shall often refer to in the following. An essential empirical consequence of bonding, it seems to us, is that the bound objects somehow stay together; they cannot recede arbitrarily: 
Finite distance condition for bonding: If certain objects $x$ s are bound to each other, then, ceteris paribus their relative spatial distance does not exceed a certain value.

The ceteris paribus clause here excludes the case that there are external forces that exceed a specific limit for the bound system in question. While there may be external forces in situations of bonding, these may not become too strong. Pushing a vase softly along a window board does not alter that the vase's parts are bound; but clashing the same vase against the wall will make it break into pieces, which are then unbound. In this sense, the finite distance condition is correct when the external forces are within a certain range. The characteristic threshold indicating the maximal strength of an external force that the bound system in question can resist before it breaks can, in principle, have any value; it indicates the robustness of the bound system. Some objects are more robust (e.g., a diamond) than others (e.g., a vase of porcelain), but it is clear that there is such a limit, even if high, for any bound system - hence the ceteris paribus clause.

Being confined to a certain spatial distance, as the finite distance criterion states, is a symptom of bonding (i.e., a necessary condition). We emphasize, however, that it is no conclusive symptom (i.e., a sufficient condition): Bodies might well be confined without being bound when external forces hold them together. A book lying on my laptop is (in the vertical dimension) constrained to follow the laptop's motion: I can lift the latter and the book will lift as well. I can lower the laptop (not too fast!) and the book will follow. However, the confinement is not due to bonding between my laptop and the book, but due to the external force that the earth exerts on both by gravitation.

When, as a result of an external force below the robustness threshold, an object of the bound system is moved, the finite distance condition implies that the other parts will move as well. When I push the cue stick against a small region of the cue ball, the complete ball will start to move. Note, however, that certain bodies let their parts some space to move, e.g., connected chain links. In these cases when one part is moved, the other parts do not move instantly. It might be the case that for a certain time one part moves while the other parts are at rest, and only when the moving part reaches the maximal distance allowed by the bonding, the other parts start to move. We can formulate these insights as follows:

Joint movability condition for bonding: If certain objects $x$ s are bound to each other, then, ceteris paribus, if one moves one of the $x \mathrm{~s}$, there is a spatial limit to this motion beyond which the other $x$ s will move as well.

This condition is a consequence of the finite distance criterion for bonding and it is a necessary condition for bound objects as well.

Now, if one assumes, as we are proposing in this paper, that one way for composition to occur ("physical composition") is that bonding occurs (plus some extra conditions), it 
is clear that these two conditions characterizing bonding directly transfer objects that compose another object due to bonding ("physical bodies"):

Finite distance condition for physical composition: If certain objects $x$ s physically compose another object $y$, then, ceteris paribus their relative spatial distance does not exceed a certain value.

Joint movability condition for physical composition: If certain objects $x$ s physically compose another object $y$, then, ceteris paribus, if one moves one of the $x \mathrm{~s}$, there is a spatial limit to this motion beyond which the other $x$ s will move as well.

Already on this operational level it seems obvious to us that this is an attractive view. For, first, requiring that the parts of composite objects stay together excludes most scattered objects right from the start (as it should be, in our view; it does not, however, rule out a certain subclass of scattered objects, which, as we shall argue, are acceptable in a scientific ontology). Second, it provides us with empirical criteria for physical composition: If two objects recede without limit or if moving the one does not move the other, the two cannot be parts of a physical composite object. Understood in this way, this endows the metaphysical concept of "physical composition" with empirical meaning. (We shall make use of these criteria in our detailed discussion of bonding and composition in Sect. 4.5,4.6, and 4.7.) Third, the conditions fit very well with common sense epistemic criteria of what counts as an object: If two objects can be moved independently, say, two (unconnected) billiard balls, they are usually not considered parts of one object.

The idea that composition is linked to joint movability goes at least back to Aristotle (1995, Metaphysics V 6, 1016a5-17). In the contemporary debate an elaborated theory based on this idea has been presented by Rosenkrantz and Hoffman, who make joint movability the core of their analysis of what it means that two objects are "joined" (1997, 84-85). Others have adopted and refined their theory (e.g., Hübner 2007, chap. II.2). In contrast, the theory that we shall develop in the following defines "bonding" by concepts from contemporary scientific theories and regards ceteris paribus joint movability only as a necessary criterion. Despite their similarity, this entails a number of differences between the two approaches, which, however, we cannot discuss in this paper.

We have already said that we regard composition by bonding as constituting what we call a "physical body". ${ }^{10}$ Here we provide a preliminary definition, which will be refined in the course of this paper (see the end of Sect. 4.7 for the result):

A physical body is an object which has physical parts that are bound together (plus some extra conditions).

${ }^{10}$ Locke calls such composite objects “mass” or "body” (1690, Essay II 27 §3). 
Typical examples for physical bodies are stones, billiard balls, tables and also nuclei, atoms and molecules. ${ }^{11}$ If one accepts the claim that every thing is part of itself, physical simples trivially count as physical bodies since the bonding condition can sensibly be required only for more than one part (but nothing hinges on this assumption for the following and we shall exclusively examine the non-trivial cases of physical bodies). By its definition, a physical body's parts fulfill the finite distant condition; and, consequently, a physical body's spatial extension is limited. Moreover, they fulfill the joint movability condition such that a physical body can be moved as a whole by moving one of its parts.

This completes our first characterization of "bonding", "composition" and "physical bodies". We are now going to develop a more detailed account of bonding that fits our scientific view of the world. This will help us refine our concepts and formulate a reliable criterion of composition.

\subsection{Bonding is not Just Interaction}

In this section we start with a negative characterization of bonding: Bonding is not just an interaction between objects, not even when the interaction is attractive. We also demonstrate that composition based on mere interaction is inappropriate: We formulate hypothetical criteria of composition based on interactions and evaluate them according to whether they guarantee the finite distance condition (for composition), whether they avoid universalism and nihilism and especially whether they yield the scientific ontology that we have just sketched (or something near enough). None of the criteria based on interaction will turn out to be appropriate. For simplicity's sake in this and the following section we shall consider interactions between only two objects; in Sect. 4.7, where we elaborate a reliable criterion based on bonding, more complex cases will be taken into account.

Van Inwagen's duplication principle demands that composition depends only upon the causal and spatiotemporal relations among the simples in question and their intrinsic properties. The current standard model of particle physics lists a total of 17 elementary

\footnotetext{
11 The definition of a physical body through bonding applies without modification to the quantum case. However, the consequence implied by that definition and the finite distance condition that a physical body's extension is limited rests on the classical assumption of definite locations for the involved objects - which is false in a standard interpretation of the quantum realm. There, objects do not have definite locations but only probabilities for being measured at certain locations; so in our reasoning one would have to replace spatial distance with probabilities of spatial distance.
} 
particle types, three of which are stable material particles: up-quarks, down-quarks and electrons. These are the fundamental building blocks of stable matter in our universe and they can influence each other by four fundamental interactions: gravitation, electromagnetism and the strong as well as the weak nuclear interaction.

Aiming at a criterion of composition that respects both the duplication principle and this ontology of modern physics, a natural choice for a principle of composition might seem to be:

\section{$\mathrm{H} 1: \exists y\left(x_{1}\right.$ and $x_{2}$ compose $\left.y\right)$ if $x_{1}$ and $x_{2}$ interact.}

In order to evaluate $\mathrm{H} 1$, consider the well-known electromagnetic forces, which can be both attractive and repulsive. Now, the fact that two like-charged objects repulse one another, plus the fact that this repulsion acts over arbitrarily large distances, ${ }^{12}$ leads to a serious objection against $\mathrm{H} 1$. Take, for instance, two electrons, which, having like charges, repulse each other. (Or if you are not familiar with elementary particles, imagine two repulsive magnets.) Placed in an idealized and otherwise completely empty universe, the electrons would repel each other until the distance between them is infinite. This process can be illustrated using a so-called potential energy graph (Fig. 4.1).

Hence, given the finite distance definition of a physical body presented above, it is clear that repulsing objects cannot count as physical bodies, for repulsing objects enlarge their distance arbitrarily save for any external forces involved. Only if there are external forces, like my hands pushing two magnets together, repulsing objects can be confined to a certain spatial region. But the external forces are relations between the allegedly composing objects and their environment and therefore violate the duplication principle (see Sect. 4.1). Therefore, in any given case of repulsing objects either the finite distance condition or the duplication principle is not met.

One obvious way to avoid these inappropriate consequences might be to limit H1 to cases of fundamental attractive interactions:

$\mathrm{H} 2: \exists y\left(x_{1}\right.$ and $x_{2}$ compose $\left.y\right)$ if $x_{1}$ and $x_{2}$ attract each other.

\footnotetext{
12 The same is not true of the other fundamental interactions: Nuclear interactions are effective only at an atomic length scale (they do not act over large distances); and while gravitation - as well as electromagnetism - influences over large distances, it is always attractive (as, e.g., between the earth and the moon).
} 


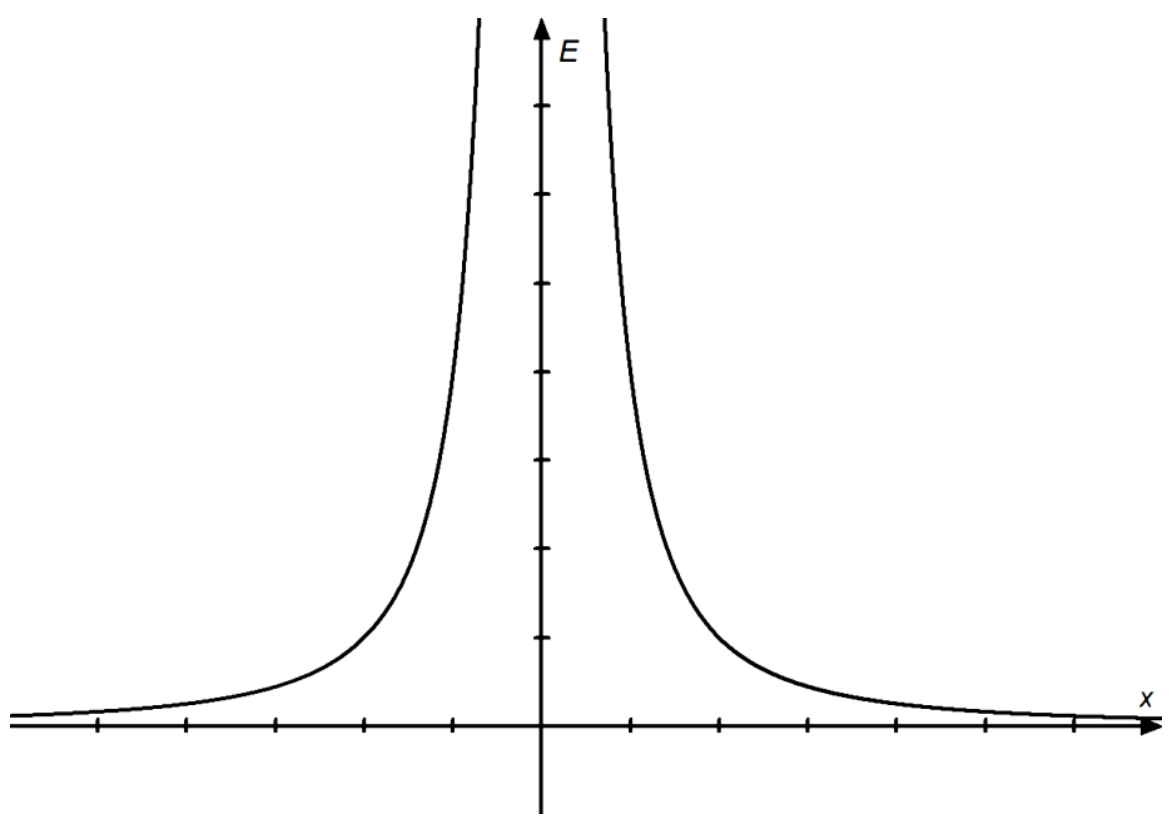

Fig. 4.1. Potential energy graph for an idealized repulsion. The $x$-axis indicates the relative distance between two objects. One is situated at the origin, while the other moves to the left or right along the horizontal x-axis. Other dimensions (up/down and front/back) are neglected. The y-axis indicates the potential energy at any given distance. It is infinite at a distance of zero and decreases with increasing distance, approaching zero only in the limit of an infinite distance. A particle in this potential field undergoes a force that points in the direction given by the negative slope. Using a suitable analogy, one can think of the graph as showing a geographic profile and of the moveable object as a ball, which is always accelerated downhill (and only in a valley feels no force).

Attraction seems to be a better candidate for a principle of composition than interactions in general (including repulsive interactions), because attracting objects, no matter how far apart, tend to reduce their distance; they tend to 'stay together' and 'stick to each other' (Fig. 4.2).

On the other hand, there are at least two serious objections to $\mathrm{H} 2$ as a principle of composition. First, the fact that gravitation acts on an infinite range implies that all massive particles attract one another. Since quarks as well as electrons are massive, according to H2, any pair of simples (and, if appropriately generalized, any collection of simples) composes an object. Interpreted as an answer to the practical version of the SCQ, H2 would suggest: "Yes, there are composed objects, but there's nothing we have to do to make any two simples compose an object. The required attraction is always there and composition always occurs in every conceivable case, without our interference." In other 
words, $\mathrm{H} 2$ leads to mereological universalism - and we have agreed with van Inwagen that universalism is not a convincing answer to the SCQ. Note that this objection to H2 on the basis of attractive interactions is also one against $\mathrm{H} 1$, which involves the attractive forces plus the repulsive ones. Moreover, in the case of H1, a similar objection can be made on the basis of electromagnetic interactions, as all quarks and electrons are charged and influence one another over arbitrary distances.

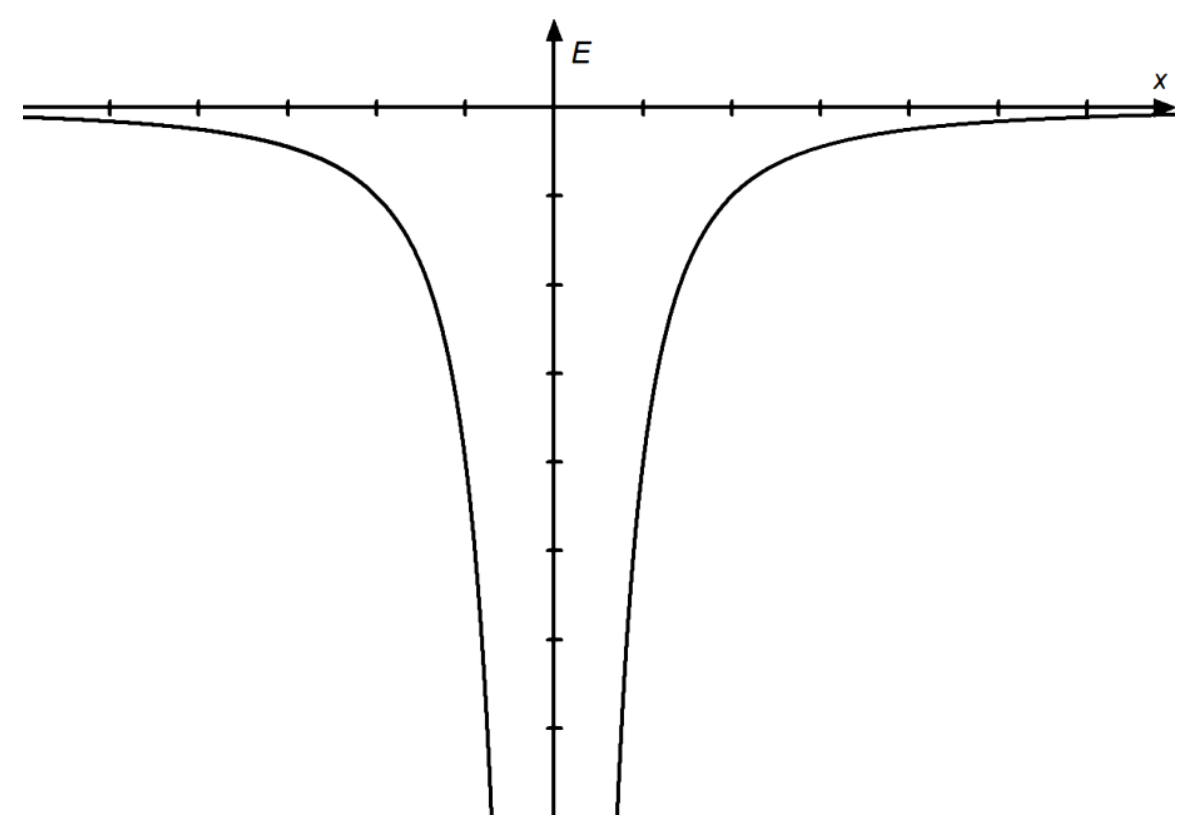

Fig. 4.2. Potential energy graph for an ideal attraction. If we place a proton and an electron, which attract another by electric force, or two planets, which attract another by gravitation, in an idealized universe, their interaction is described by the graph shown here. Since the slope of the graph (as we have explained in fig. 4.1) is a measure for the force, the graph indicates an attractive force in direction towards the origin that gets the stronger the closer the objects are. The graph having nowhere slope zero indicates that attraction is present at arbitrary distances. According to the suggestive analogy we have introduced, a ball at rest placed on a slope of this form will always start to roll towards the center, ever accelerating (until it has reached the center).

Secondly, while attraction is a tendency to make objects stay close to one another, it is no guarantee. Consider an asteroid approaching the sun. Due to gravitational attraction, the sun deflects the asteroid from its original straight path, but the asteroid's motion is so fast that it escapes the sun's gravitational field and disappears (on a hyperbolic trajectory) into the depths of the universe. Do the asteroid and the sun compose something during the 
time of their mutual attraction? Surely not according to our finite-distance criterion. There are many such cases in which the strength of attraction does not suffice to keep several objects within a limited distance. (A similar scattering process occurs when an electron rapidly passes by a proton.) The reason is that objects have kinetic energy as they move through space, and this kinetic energy can outweigh the confining potential energy. It is important to note that kinetic energy is an intrinsic property of physical objects; it is therefore consistent with the duplication principle that kinetic energy plays a role for the evaluation of composition. So while gravitation and electromagnetism do make every pair of massive or unlike-charged objects attract one another, this does not result in all these pairs of objects staying close to one another. Consequently, H2 in some cases violates the finite-distance condition - which makes it unsuitable for judging composition.

\subsection{Bonding as a Relation Between Kinetic and Potential Energy}

The story about kinetic energy outweighing potential energy provides a lesson about the scientific nature of bonding, which we shall explain in this section. The lesson is best illustrated with the graphs we have introduced. Consider an object in a gravitational potential (Fig. 4.3). As an example, one can think of an asteroid in the sun's gravitational field. The object in the potential, the asteroid, is represented by a horizontal line indicating its total energy level. (An object's total energy is the sum of its potential and kinetic energy.) Depending on this energy level, the asteroid is either bound or free. In the present diagram it is the line of zero energy that is the hallmark of bonding or non-bonding (According to a widespread convention, energy zero is chosen at the maximum of the potential energy graph.) If the asteroid's energy level lies above this line, its total energy outweighs the potential barrier. Then, though the sun attracts the asteroid, the latter's motion is not confined to a finite region: It will move to infinity (though, due to the sun's attraction, on a hyperbolic trajectory). The non-confinement is represented by the fact that the horizontal energy line does not intersect with the potential graph, i.e., there is no obstacle to the motion of the asteroid at any point. Such states are usually called "unbound" or "free".

In contrast, below the line of zero energy, the asteroid is bound in the sun's gravitational field. This is represented by the fact that at each side the corresponding horizontal energy lines are limited by an increasing potential energy function ("potential walls" or "potential barriers"). This configuration of two potential walls delimiting a spatial region is called "potential well". Being situated in a potential well of the sun's gravitational field means that the asteroid's motion is confined to the region between the delimiting walls. In these cases the asteroid might orbit the sun (on an elliptic trajectory) with a certain 
maximal distance. The strength of the binding, the binding energy, is given by the difference between the horizontal line representing the bound object and the line of zero energy: the larger the difference, the stronger the binding; the difference equals the minimal amount of external energy that is needed to separate the two objects infinitely.

So far we have made clear that an object is in a bound state when its total energy is lower than the potential barrier. (If the potential barriers on each side are unequal, it needs to be lower than the lowest barrier in order to be bound.) We still have to make clear in what sense this means that in such cases potential energy outweighs kinetic energy. This can be seen as follows. If, as we have assumed here, the point of zero energy is set to the height of the lowest potential barrier, being bound means to have a negative total energy $\left(E_{\mathrm{t}}<0\right)$. And since the total energy of an object is the sum of its kinetic and potential energy $\left(E_{\mathrm{t}}=E_{\mathrm{k}}+E_{\mathrm{p}}\right)$, this implies that the kinetic energy must be smaller than the negative potential energy $\left(E_{\mathrm{k}}<-E_{\mathrm{p}}\right)$. It is in this sense (with the minus sign in place) that negative potential energy has to outweigh kinetic energy in order for bonding to occur. Or, vice versa, in order for an object to be unbound $\left(E_{\mathrm{t}}>0\right)$, kinetic energy has to outweigh negative potential energy $\left(E_{\mathrm{k}}>-E_{\mathrm{p}}\right)$. The relation between kinetic and potential energy that determines whether an object is either bound or unbound can also be seen in Fig. 4.3. Note that while kinetic energy is always positive, potential energy is negative when the potential graph is below the zero energy line.

This discussion suggests the following definition:

Bonding: A physical object $x_{1}$ is bound to another $x_{2}$ if and only if

(i) $\quad x_{2}$ creates a potential well (of a kind that affects $x_{1}$ ) in every spatial dimension, and

(ii) $\quad x_{1}$ is located inside the potential well, and

(iii) $x_{1}$ 's total energy is lower than the minimal height of the potential well.

By what we have said above, condition (iii) is equivalent to the fact that $x_{1}$ 's negative potential energy outweighs its kinetic energy $\left(E_{\mathrm{k}}<-E_{\mathrm{p}}\right)$ given that the point of zero energy is chosen at the minimal height of the potential well.

This definition of bonding clearly implies the finite distance condition: If an object $x_{1}$ is bound to another $x_{2}$ in the indicated way, its motion is confined by the potential well and thus the two objects' relative spatial distance is limited. Furthermore, the definition implies that moving $x_{2}$ will move $x_{1}$ since moving $x_{2}$ will shift the potential well, which in turn will shift $x_{1}$ when it hits one of the walls (as long as the motion is not as fast as to let $x_{1}$ slip over the potential barrier). So the joint movability condition is fulfilled as well. 


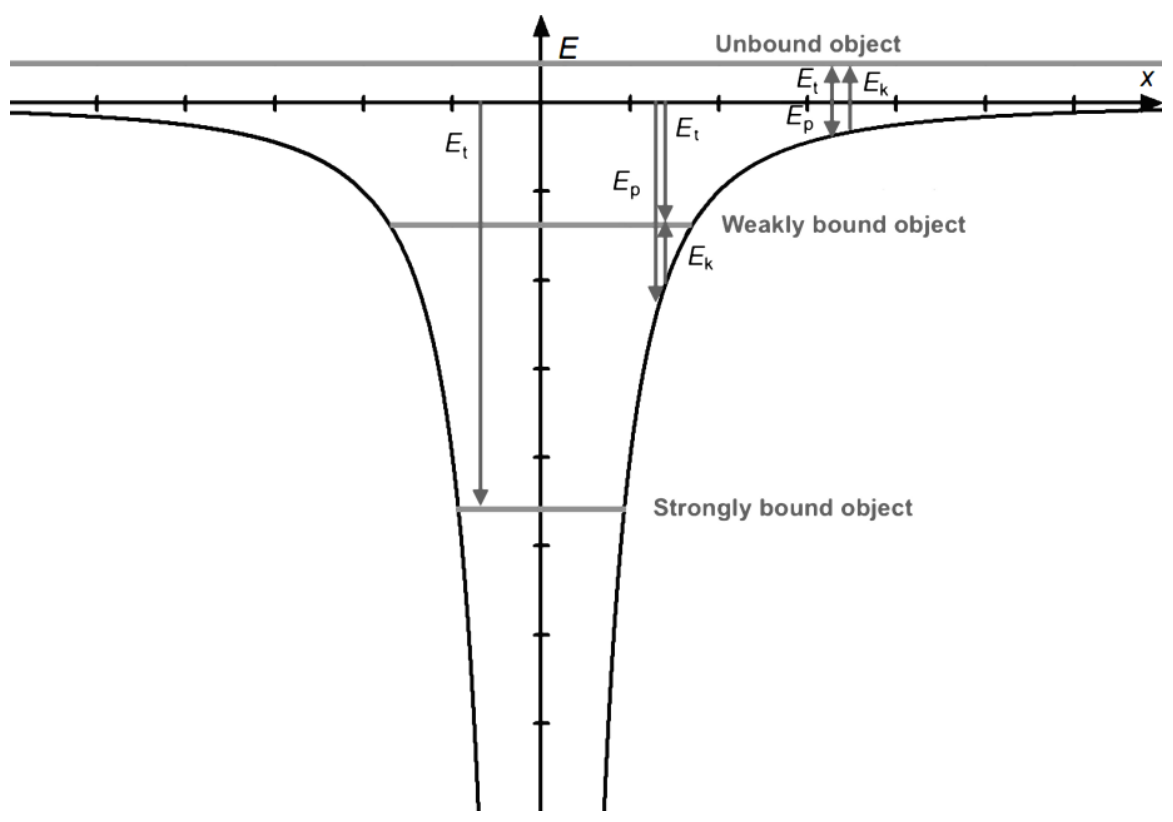

Fig. 4.3. Unbound and bound objects in a potential well. The unbound object (e. g. an asteroid on a hyperbolic trajectory) has sufficient kinetic energy to escape the attraction well and recede infinitely. In contrast, the motion of both bound objects (e.g. asteroids on elliptic trajectories) is restricted to the area between the potential walls. As the strongly bound object has less kinetic energy than the weakly bound object, its energy line is situated lower on the energy axis, resulting in a stronger spatial confinement (horizontal length of the line). The vertical distance between the energy line of a bound object and the x-axis is the energy that is needed, to unbound an object; it is called the binding energy $\left(\mathrm{E}_{\mathrm{b}}\right)$. The figure also illustrates the relation between total energy $\left(\mathrm{E}_{\mathrm{t}}\right)$, potential energy $\left(E_{p}\right)$ and kinetic energy $\left(E_{k}\right)$; arrows pointing upwards denote positive energies, those pointing downwards negative ones.

Besides spatial delimitation and joint movability, bonding has another important feature, namely that a bound system is stable. Minor interventions in a bound system - i.e., interventions that do not provide enough energy to hurdle the potential barrier - do not suffice to unbind the objects involved. Instead, the system either returns to its original state or establishes a new stable state. Again, an asteroid on an elliptic trajectory serves as an example. If it was rather weakly pushed towards the sun or deflected by the gravitation of a third object, it would swing into a new stable orbit around the sun. Neither would the asteroid collide with the sun, nor would it escape the sun's gravitation. Therefore, bonding gives rise to stable physical states, which are suitable to be identified with composite physical objects - the kind of objects we are looking for in our ontology. 
Note that while in this section so far we have discussed exclusively astronomical objects with gravitational bonding, bonding can equally be due to electromagnetic forces or strong nuclear forces. Moreover, real objects need not be composed of point-like objects as we have assumed up to now. In Fig. 4.4 we present the potential well for an extended object.

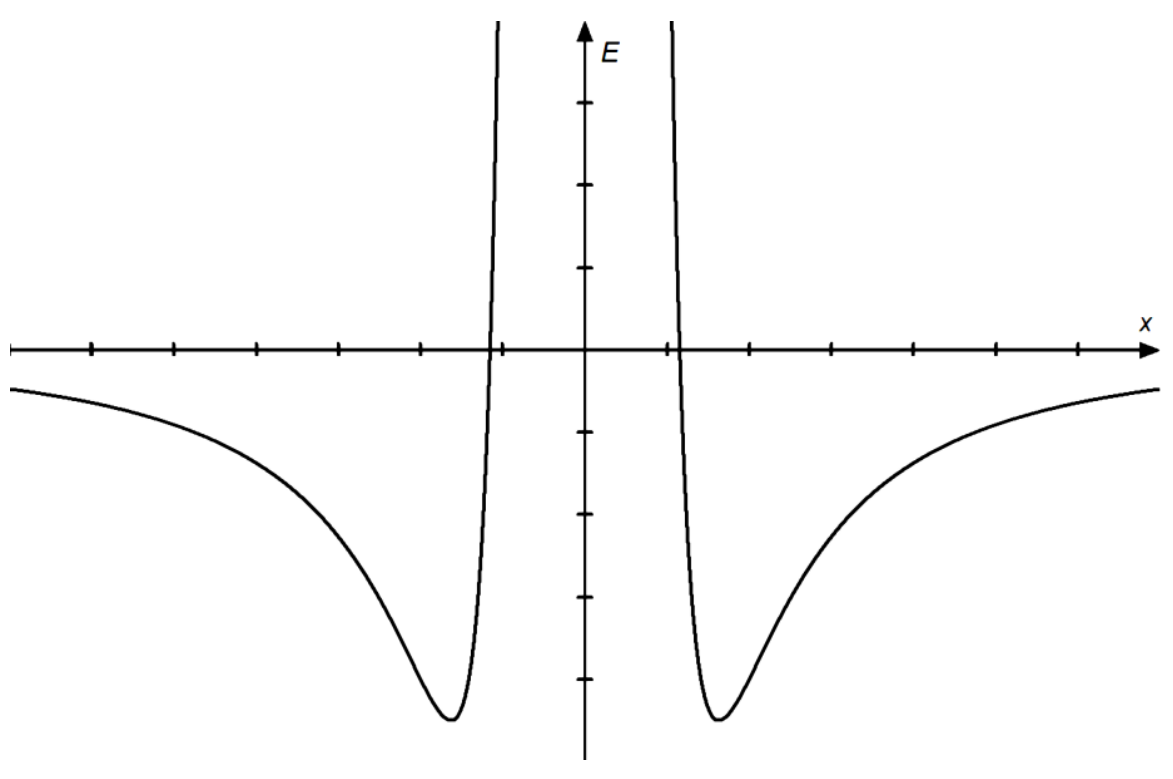

Fig. 4.4: Potential well of an extended object. In contrast to the potential well of an ideal point particle (see fig. 4.3) the potential well of real, extended, attractive objects are not attractive at all points; and they are not infinitely deep. While at larger distances attractive forces prevail, at short distances strong repulsion due to the impenetrability of matter dominate. For unlikely charged atoms this is known as the Lennard-Jones-Potential (but a similar potential holds for gravitational interactions of extended objects; in both cases the short-range repulsion is due to electromagnetic forces and the Pauli exclusion). In contrast to the case of point particles, the potential well here is not infinitely deep, but rather has a minimum at a certain distance. The minimum allows for static binding, i.e. situations where two particles bind without moving relative to another.

Bonds are established by many different processes. A prominent example is chemical reactions unifying two molecules into a single larger one by means of chemical bonding. Before the reaction the smaller molecules can move freely and recede arbitrarily. During the chemical reaction, however, they become parts of a larger molecule and their motion (relative to each other) is henceforth closely confined by potential walls. Another exam- 
ple is gluing two objects together. The glue either chemically binds to each of the two joined objects or leads to intermolecular bonding with each object.

So far we have only considered the most obvious type of bonding - bonding by attraction. In these cases, the confining potential walls are due to an attractive interaction between the involved objects. Bonding by attraction, however, is not the only mechanism to accomplish spatial confinement. Bonding can also be achieved by repelling the object in question at the borders of a spatial region. Our definition comprises such cases as well, as it only refers to the existence of a potential well, without specifying that the well is due to attractive interactions. We call such cases bonding by repulsion.

A simple example for bonding by repulsion is a ball in a solid box, which can move freely inside the box but is repelled when it hits one of the walls. The repulsion is due to the impenetrability of matter: Solid bodies consist of atoms or molecules, which are surrounded by clouds of electrons; since these clouds are negatively charged (and also due to the Pauli exclusion principle), they act strongly repulsively when two objects approach each other. (In principle this repulsion acts over any distance, but it becomes extremely weak when the two bodies are not very close to each other; and this is the reason why this repulsion is associated with two bodies in contact.) The impenetrability of matter is the most common source of bonding by repulsion.

On a technical level, repulsion due to impenetrability is described by potential walls as well. Again, for the sake of simplicity, we take into account only one dimension of the ball's motion: Each of the box's impenetrable material walls creates a repulsive potential wall (similar to the one in Fig. 4.1 but steeper), and the total potential graph is the sum of the two resulting in a potential well that confines the motion of an object between the walls (Fig. 4.5). This illustrates that, besides by attraction, a potential well, and hence bonding, can be generated by repulsive interactions as well. Further examples for this kind of bonding are the following: screwing or nailing objects to each other, stapling sheets of paper, pinning a poster to a wall, putting objects in closed containers, building a Lego house, fixing an object in a vise or mooring a ship to a bollard. 


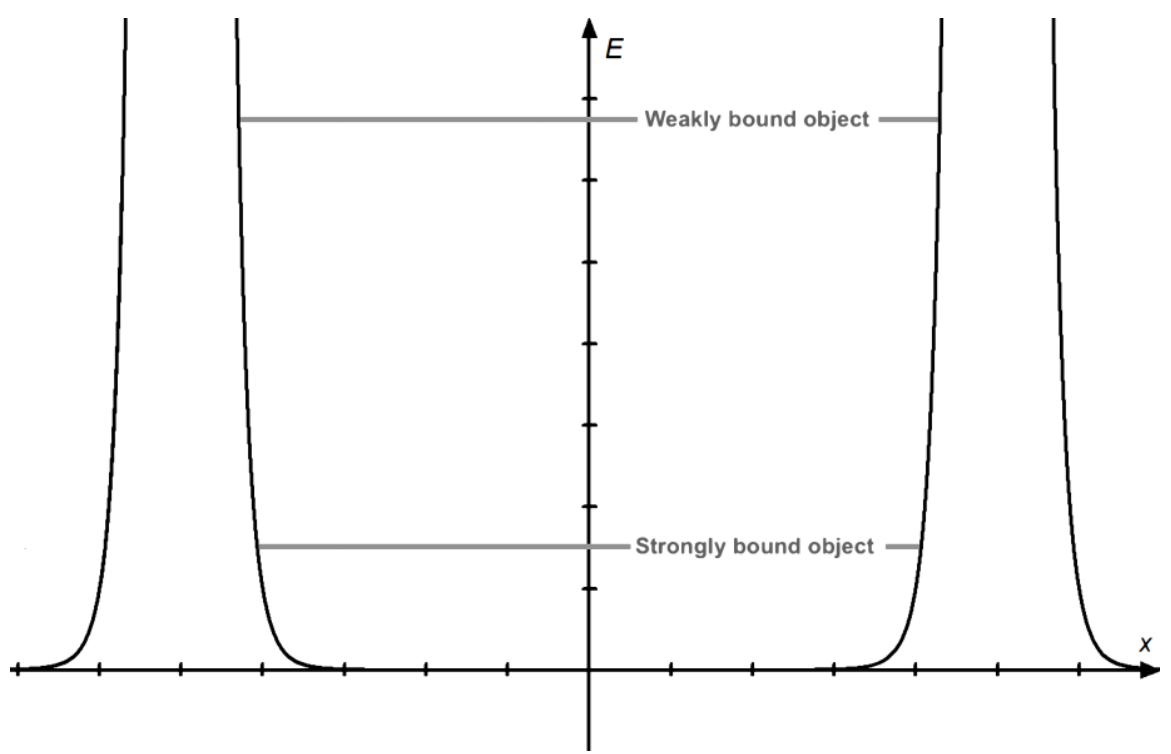

Fig. 4.5. Bonding by repulsion. An object in a two-fold repulsive potential, with peaks at the origin and at $\mathrm{x}=2$. One might think of a charged ball in a likely charged box. The object placed anywhere between the repulsive walls is bound by these walls and its motion is confined to a certain region. The region of confinement is the larger, the larger the kinetic energy of the object is.

This section concludes our scientific characterization of bonding. While in Sect. 4.3 we characterized bonding in terms of the finite distance condition as well as the joint movability condition, here we have seen that these features are implied when the kinetic and potential energy of a system relate in a certain way: An object is bound when there are potential barriers in all spatial directions and these barriers are higher than the object's total energy. Or, equivalently, if the point of zero energy is chosen at the height of the lowest potential barrier, negative potential energy has to outweigh positive kinetic energy. Then, ceteris paribus, the barriers constrain the possible motions of the object (finite distance condition); and this implies joint movability with the other object(s) that generate(s) the potential barriers. This general characterization of physical bonding both comprises the case of bonding by attraction as well as bonding by repulsion. 


\subsection{Composition by Bonding}

Having clarified the concept of bonding in scientific terms, we now have to discuss suitable criteria of composition based on bonding. Let us start by considering the following hypothesis:

H3: $\exists y\left(x_{1}\right.$ and $x_{2}$ compose $\left.y\right)$ if $x_{1}$ and $x_{2}$ bind with one another.

Clearly, this proposal suffers from the fact of involving only two parts, and it has to be generalized to arbitrarily many binding objects. In objects with more than two parts it is typically the case that not all parts bind directly with one another; some only bind indirectly. Take, for instance, a rather small molecule like ethane $\left(\mathrm{C}_{2} \mathrm{H}_{6}\right)$. In an ethane molecule the two carbon atoms directly bind with each other, and each carbon atom binds with three hydrogen atoms. In Fig. 4.6 $\mathrm{C}^{(\mathrm{I})}$ is directly bound with $\mathrm{C}^{(\mathrm{II})}$ as well as $\mathrm{H}^{(1)}, \mathrm{H}^{(2)}$ and $\mathrm{H}^{(3)}$. But the remaining three hydrogen atoms $-\mathrm{H}^{(4)}, \mathrm{H}^{(5)}$ and $\mathrm{H}^{(6)}$ - are not directly bound to this carbon atom. Additionally, $\mathrm{H}^{(4)}$ is not directly bound to $\mathrm{H}^{(5)}$ and so on.

Fig. 4.6. Chemical structure of ethane. The atoms are numbered in order to simplify the reference to certain atoms (the numbers do not indicate any chemical properties).

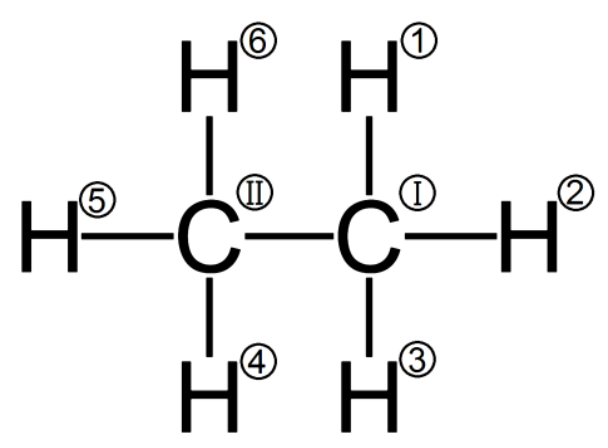

In order to capture such cases, we generalize H3 to arbitrarily many $x$ s and allow both for direct and indirect bonding:

H4: $\exists y$ (the $x$ s compose $y$ ) if every pair of the $x$ s binds directly or indirectly.

Let us characterize indirect bonding more precisely. It rests on the assumption that the bonding relation is transitive: If $\mathrm{A}$ is bound with $\mathrm{B}$ and $\mathrm{B}$ is bound with $\mathrm{C}$, it follows that $\mathrm{A}$ is bound with $\mathrm{C}$ - though $\mathrm{A}$ and $\mathrm{C}$ are not directly bound; they are only bound indirectly, namely via B. If several objects are indirectly bound, it is possible to reach any of them following an uninterrupted chain of direct bonds. This holds no matter which object 
is chosen as a starting point. It is, of course, possible that two parts are connected by more than one chain of bonds.

$\mathrm{H} 4$ implies that a whole molecule is an object composed of indirectly bound atoms, but so are any coherent subsets of this molecule. Nothing in H4 demands that we may consider only a complete structure of objects bound to one another as a composite object. Hence, H4 implies the existence of so-called "arbitrary undetached parts" (van Inwagen 1981), like the handle of a coffee mug or the first 27 pages of this book. Since, like van Inwagen, we would like to avoid such arbitrary undetached parts, which would blow up the ontology, we have to add a further criterion preventing the existence of arbitrary coherent substructures:

H5: $\exists y$ (the $x$ s compose $y$ ) if

(a) every pair of the $x$ s binds directly or indirectly and

(b2) there is no extension of the $x$ s that fulfills (a). ${ }^{13}$

(Note that here the unusual labelling, to have (b2) without (b1), is intended because in following revisions of the principle we will have to add another condition (b1) between (a) and (b2).) The additional criterion (b2) makes sure that we exclusively grasp the whole binding structure and not just a part of it. If someone claimed that the handle of a coffee mug alone composed an object, his definition would meet condition (a) while violating condition (b2): Each pair of the handle's molecules do bind directly or indirectly, but there is an extension of these molecules which also fulfills (a). ${ }^{14}$ According to H5, all bound parts have to be included in the $x \mathrm{~s}$ in order to meet condition (b2), and in the case of the handle it is only the whole mug that fulfills this criterion.

While H5 successfully rules out arbitrary undetached parts, it runs into the opposite extreme by requiring composite objects to be too large. For instance, objects that stand on the earth's surface, like a coffee mug, are gravitationally bound to the earth, and any movement of the earth thus causes a movement of the coffee mug. So according to H5, the coffee mug is not a whole, but only a part of a larger system comprising the mug and the earth. But the earth plus mug in turn is not a whole either, but a part of the solar system since it is gravitationally bound to the sun, and the sun binds with other planets, their

\footnotetext{
${ }^{13}$ Precisely, H5 reads: Let the variably $y$ rande over all objects, $x_{1}$ and $x_{2}$ over the $x \mathrm{~s}$, and $z$ over all objects except the $x$ s. Then: $\exists y$ (the $x$ s compose $y$ ) if (a) $\forall x_{1} \forall x_{2}\left(x_{1} \neq x_{2}\right) \rightarrow x_{1}$ and $x_{2}$ bind directly or indirectly) and (b2) $\sim \exists z \exists x_{1}$ ( $z$ and $x_{1}$ bind directly).

14 Note that the handle satisfies both the finite distance and the joint movability condition for physical objects. Thus, it becomes apparent that these conditions are only necessary and not sufficient for physical composition.
} 
moons and so on. In this way, according to H5, besides the simples, only a small number of very large composite objects exists. It might even be the case that this hypothesis amounts to the existence of only one composite object - the whole universe - in case everything in the universe is somehow bound to one another. This ontology, however, is much too undifferentiated for the scientific picture we are aiming at. ${ }^{15}$

These undesired consequences can be avoided by taking into account the fact that bonding comes in degrees. A proton and a neutron in a nucleus bind more strongly with one another than two atoms in a molecule, and two atoms are more strongly bound than two molecules. These differences in binding strength can be quantified by differences in binding energy: The binding energy is the energy that is needed to separate two bound objects from each other to infinite distance. Defined in this way, binding energies have positive values and indicate that a bond is stronger, the greater its binding energy is. ${ }^{16} \mathrm{~A}$ common unit for binding energy is $\mathrm{kJ} / \mathrm{mol} .{ }^{17}$ Characterizing bonds by binding energies allows to discriminate between bindings of different strengths: We can define that a certain composite object includes all and only those objects that are bound to one another with a binding energy that is larger than a certain minimal threshold. We can integrate this specification into the composition criterion as follows:

H6: $\exists y$ (the $x$ s compose $y$ ) if

(a) every pair of the $x$ s binds directly or indirectly and

(b) there is an amount of energy $e>0$ such that (b1) all direct bonds between the $x$ s have an energy greater than or equal to $e$ and (b2') there is no extension of the $x$ s that fulfills (a) and (b1). ${ }^{18}$

By condition (b), hypothesis H6 introduces an energy threshold $e$ : According to (b1), all objects that bind more strongly than or equal to $e$ are included in the composite object

\footnotetext{
${ }^{15}$ But cf. Schaffer (2010) who defends an even more radical monism on the basis of quantum entanglement (which we have said to neglect here): He defends the thesis that there literally is only one object, the whole universe. So unlike the monism threatening from H5, Schaffer's monism even denies the existence of simples.

${ }^{16}$ If the point of zero energy is chosen at the maximum of the lowest potential barrier, the binding energy equals the negative total energy of a system.

17 The joule is the basic unit for measuring energy and " $\mathrm{kJ}$ " means " 1000 joules"; specifying the binding energy per mole means to give the binding energy for $6.022 \times 10^{23}$ bonds of the same type, i.e. $1 \mathrm{~kJ} / \mathrm{mol}=$ $6.022 \times 10^{-20} \mathrm{~J}$.

${ }^{18}$ Here is H6 in a precise form: Let the variable $y$ range over all objects, $x_{1}$ and $x_{2}$ over the $x \mathrm{~s}, z$ over all objects except the $x$ s, and $e$ over possible energy values. Let us furthermore assume that if two objects bind directly, they are not identical. Then: $\exists y$ (the $x$ s compose $y)$ if (a) $\forall x_{1} \forall x_{2}\left(x_{1} \neq x_{2} \rightarrow x_{1}\right.$ and $x_{2}$ bind directly or indirectly) and (b) $\exists e\left[(\mathrm{~b} 1) \forall x_{1} \forall x_{2}\right.$ ( $x_{1}$ and $x_{2}$ bind directly $\rightarrow$ binding energy $\left(x_{1}, x_{2}\right) \geq e$ ) and (b2') $\neg \exists z \exists x_{1}$ ( $z$ and $x_{1}$ bind directly $\&$ binding energy $\left(z, x_{1}\right) \geq e$ )].
} 
in question, while all objects binding more weakly are excluded. Cutting off all bonds that are below a certain strength solves the problem of H5 that only huge composite objects exist. Depending on the threshold $e$, objects can be rather small (if the threshold is high) or large (if the threshold is low). It is important to note that H6 does not assume one single threshold (which would be arbitrary), but that $e$ can and does take any value: For each possible value of $e$ and for each collection of $x \mathrm{~s}$, H6 potentially defines a composite object.

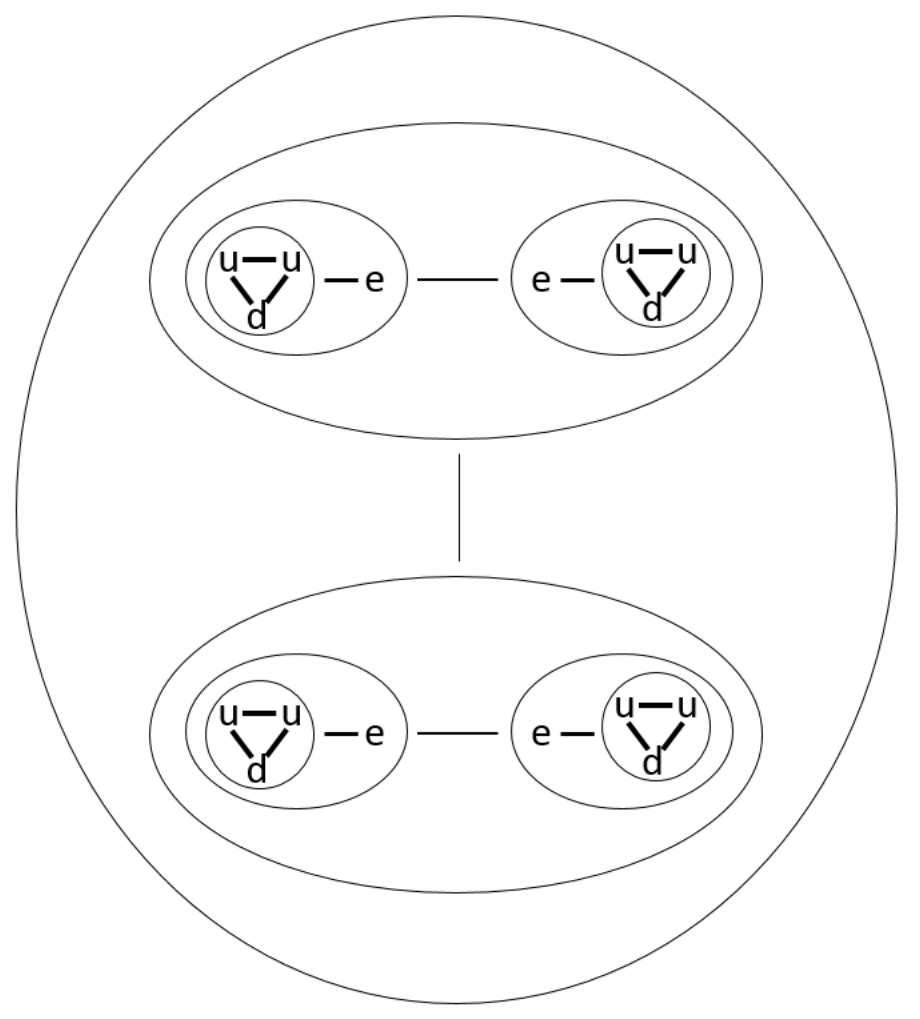

Fig. 4.7. A world of two $\mathrm{H}_{2}$ molecules. Two up quarks (u) and one down quark (d) compose a proton, respectively; a proton and an electron (e) constitute a hydrogen atom; two hydrogen atoms form a hydrogen molecule. Furthermore, assuming that the temperature of the present universe is very low, the hydrogen atoms bind (by van der Waal forces) and form an H2-H2 compound. Lines represent bonds and the thickness of each line represents the strength of the corresponding bond. Circular or oval shapes represent composite objects. 
In order to illustrate the consequences of $\mathrm{H} 6$, consider a world with only two $\mathrm{H}_{2}$ molecules (Fig. 4.7). On a fundamental level, the level of simples, there exist eight up quarks, four down quarks and four electrons. Which composite objects exist in this world according to H6? The only thing one has to do to answer this question is to let $e$ take on all possible values and to note the objects that result at these values. At very high values of $e$, no composite objects are implied because none of the bonds in this world is stronger than these high values. Lowering the value of $e$ to $8.97 \times 10^{10} \mathrm{~kJ} / \mathrm{mol}$, which corresponds to the binding energy of two up quarks and one down quark forming a proton, yields the existence of four protons (for an overview of some binding energies see Table 1). For a large range of lower values of $e$, nothing new happens. Only when $e$ reaches the value $1.31 \times 10^{3} \mathrm{~kJ} / \mathrm{mol}$, which is the binding energy between a proton and an electron forming a hydrogen atom, are four hydrogen atoms implied by H6. And only when $e$ reaches the value $436 \mathrm{~kJ} / \mathrm{mol}$, which is the binding energy of the two hydrogen atoms in an $\mathrm{H}_{2}$ molecule, do the hydrogen molecules enter the scene. So besides the simples, we have the protons, the hydrogen atoms and the hydrogen molecules in our ontology - just as any scientist would assume. And if the temperature in that universe were very low, say below $21 \mathrm{~K}$, the van der Waals interaction between the hydrogen molecules outweighs their kinetic energy such that the molecules are bound to one another as well. So when $e$ reaches the energy of this van der Waals bond (somewhere below $5 \mathrm{~kJ} / \mathrm{mol}$ ), H6 would imply the existence of the $\mathrm{H}_{2}-\mathrm{H}_{2}$ compound as well (which were the complete universe in the present case). It is clear that in worlds with many simples, H6 would also generate mid-sized and macroscopic objects that we are used to. In this way, H6 generates the familiar scientific ontology that we have been aiming at.

While H6 leads to a large number of objects in the universe, it is important to note that the implied ontology is nevertheless far from mereological universalism. For composition by bonding, as we suggest it here, still precludes objects with unbound parts, even if the threshold is set very low. Very weak indirect bonding can lead to huge ramified objects, but, nevertheless, there always has to be a path of bonding between all parts of these objects.

We would like to emphasize that, according to our criterion H6, all objects exist in the same sense no matter how low or high the threshold $e$ for the respective object is. Especially, we do not claim that an object's existence is a gradual phenomenon. For any threshold, all objects that bind more strongly than the threshold, the composite system exists. Nor do we claim that existence is a vague matter: The criterion given by the thresh- 
old is sharp and so is our ontology. In this way we avoid all logical and ontological problems entailed by gradualness or vagueness of existence. ${ }^{19}$

There is, however, also a clear sense in which objects with low or high thresholds differ: Objects with a high threshold are robust, while objects with a low threshold have to count as fragile. Bonding energy is an exact way to theoretically grasp this difference. We define the binding energy of the weakest bond of a composite object as the robustness of that object. ${ }^{20}$ This seems to be an appropriate definition for measuring the stability of an object, because it is, of course, the weakest element in a chain that determines the strength of the whole chain.

There remains a last shortcoming of $\mathrm{H6}$ that we have to adjust. The criterion yields a composite object, say, a hydrogen atom, for any value of $e$ that is lower than or equal to the binding energy in question (in the case of the hydrogen atom: the binding energy between proton and electron) and greater than the next lower binding energy (in the present example: the binding energy between the two hydrogen atoms that form the hydrogen molecule). This would yield infinitely many similar composite objects (hydrogen atoms) with the very same parts where only one composite object should be composed. In order to avoid this artificial explosion of entities, we suggest the following adaption that finally yields the criterion of physical composition that we want to suggest:

Criterion of physical composition: $\exists y$ (the $x$ s compose $y$ ) if

(a) every pair of the $x$ s binds directly or indirectly and

(b) there is an amount of energy $e>0$ such that

(b1) all direct bonds between the $x$ s have an energy greater than or equal to $e$ and

(b2') there is no extension of the $x$ s that fulfills (a) and (b1) and

(b3) $e$ is the maximal amount of energy for which (b1) is true. ${ }^{21}$

\footnotetext{
${ }^{19}$ Evans (1978) presents, on a single page, a formal reductio argument showing that the assumption of vague existence is self-contradictory. In contrast, van Inwagen (1990, chap. 17) defends this idea. For an extensive investigation into the problem see Mancin (2012).

${ }^{20}$ Precisely: Let the variables $x_{1}$ and $x_{2}$ range over the $x \mathrm{~s}, e$ and $e$ over possible energy values. An object $a$, which is composed by the $x \mathrm{~s}$, has robustness $e$ if and only if (a) $\exists \mathrm{e}$ [(b1) $\forall x_{1} \forall x_{2}$ ( $x_{1}$ and $x_{2}$ bind directly $\rightarrow$ binding energy $\left.\left(x_{1}, x_{2}\right) \geq e\right)$ and (b3) $\forall e^{\prime}\left(e^{\prime}>e \rightarrow \exists x_{1} \exists x_{2}\left(x_{1}\right.\right.$ and $x_{2}$ bind directly \& binding energy $\left(x_{1}, x_{2}\right)<$ $\left.\left.e^{\prime}\right)\right)$ ].

${ }^{21}$ Formally: Let the variable $y$ range over all objects, $x_{1}$ and $x_{2}$ over the $x \mathrm{~s}, z$ over all objects except the $x \mathrm{~s}$, and $e$ and $e$ ' over possible energy values. Let us furthermore assume that if two objects bind directly, they are not identical Then: $\exists y$ (the $x$ s compose $y$ ) if and only if (a) $\forall x_{1} \forall x_{2}\left(x_{1} \neq x_{2} \rightarrow x_{1}\right.$ and $x_{2}$ bind directly or indirectly) and (b) $\exists e$ [(b1) $\forall x_{1} \forall x_{2}\left(x_{1}\right.$ and $x_{2}$ bind directly $\rightarrow$ binding energy $\left(x_{1}, x_{2}\right) \geq e$ )) and (b2') $\exists z \exists x_{1}\left(z\right.$ and $x_{1}$ bind directly \& binding energy $\left.\left(z, x_{1}\right) \geq e\right)$ ) and (b3) $\forall e^{\prime}\left(e^{\prime}>e \rightarrow \exists x_{1} \& \exists x_{2}\left(x_{1}\right.\right.$ and $x_{2}$ bind directly \& binding $\left.\left.\left.\operatorname{energy}\left(x_{1}, x_{2}\right) \geq e^{\prime}\right)\right)\right]$.
} 
Table 4.1: Examples for typical binding strengths.

\begin{tabular}{|c|c|}
\hline Type of bonding & Binding energy in $\mathrm{kJ} / \mathrm{mol}$ \\
\hline \multicolumn{2}{|l|}{ Nuclear bonds } \\
\hline Quarks within a proton ${ }^{a}$ & $8.96 \times 10^{10}$ \\
\hline Proton-neutron bond in deuterium ${ }^{b}$ & $2.15 \times 10^{8}$ \\
\hline \multicolumn{2}{|l|}{ Intra-atomic bonds } \\
\hline Proton-electron bond in $\mathrm{H}$-atom ${ }^{\mathrm{c}}$ & $1.31 \times 10^{3}$ \\
\hline \multicolumn{2}{|l|}{ Interatomic bonds } \\
\hline $\mathrm{N}_{2}$ triple covalent bond ${ }^{\mathrm{d}}$ & 945 \\
\hline $\mathrm{Na}^{+} \mathrm{Cl}^{-}$ionic bond ${ }^{e}$ & 584 \\
\hline $\mathrm{H}-\mathrm{H}$ covalent bond ${ }^{\mathrm{f}}$ & 436 \\
\hline $\mathrm{C}-\mathrm{H}$ covalent bond ${ }^{\mathrm{g}}$ & 412 \\
\hline $\mathrm{C}-\mathrm{C}$ covalent bond ${ }^{\mathrm{h}}$ & 348 \\
\hline \multicolumn{2}{|c|}{ Intermolecular bonds } \\
\hline Hydrogen bridge bond in water ${ }^{\mathrm{i}}$ & 19 \\
\hline Van der Waals bonds & $\sim 0-5$ \\
\hline
\end{tabular}

Indicating the energy required to break a certain bond, the bond-dissociation energy is a common measure for the strength of a bond. As is usual, the energies are given for 1 mole $\left(=6.022 \times 10^{23}\right)$ of bound objects. Bonds of a similar strength are grouped together. The higher the value of the binding energy, the stronger is the bond.

${ }^{a}$ This value is a rough estimate, which indicates the difference between the rest mass of the proton and the rest mass of its three constituent quarks (see Povh et al. 2015, 133, 265). This energy is carried by sea quarks and gluons. Unlike typical binding energies it does not indicate the amount of energy required to separate the bound objects: Due to "confinement" isolating quarks is impossible.

b Povh et al. 2015, 292.

${ }^{\mathrm{c}}$ Ionization enthalpy, see Atkins and de Paula 2011, 354.

${ }^{d}$ Dissociation enthalpy, see Atkins and de Paula 2011, 51

e Single ionic bond between nearest neighbors in $\mathrm{NaCl}$ lattice, see Christen and Meyer 1997, 105. Note that the stability of an $\mathrm{NaCl}$ crystal depends on interactions of each atom with all surrounding atoms (including attractive and repulsive interactions), which yields a lattice energy of $788 \mathrm{~kJ} / \mathrm{mol}$.

${ }^{\mathrm{f}}$ Dissociation enthalpy, see Atkins and de Paula 2011, 51.

g Average dissociation enthalpy, see Atkins and de Paula 2011, 51.

${ }^{\mathrm{h}}$ Average dissociation enthalpy, see Atkins and de Paula 2011, 51.

${ }^{i}$ See Atkins and de Paula 2011, 434.

${ }^{j}$ The energy of van der Waals bonds depends on the exact type of interaction and molecules involved as well as the distance of the molecules; see Atkins and de Paula 2011, 424-433. 
Condition (c) guarantees that only for the value $e$ that equals the lowest binding energy between the $x \mathrm{~s}$ is the existence of the composite object implied. For instance, only for the value of $e$ that equals the binding energy between the proton and the electron does the existence of the hydrogen atom follow. For all values of $e$ that are smaller than this binding energy and greater than the next lower binding energy (the binding energy of the hydrogen atoms), the existence of the hydrogen atom is not implied any more. And this is how it should be in order to get the scientific ontology we have been aiming at. The value of $e$ for which the composite object is implied, then equals the strength of the weakest bond, i.e. the robustness of the object.

This concludes our characterization of composition by bonding. Here we have qualified the claim that binding objects compose another object. We have (a) discerned between direct and indirect bonding; (b2) in order to avoid arbitrary undetached parts, we have required composite objects to be maximal collections of bound objects; (b1) we have introduced a threshold for (b3) the minimal binding energy in an object, which gives the object's robustness. These four conditions are summarized in our criterion of physical composition. It remains to say that by this criterion we can also provide the precise definition of a physical body that we could only sketch in Sect. 4.3:

A physical body is an object whose parts, the $x$ s, are physical objects and

(a) every pair of the $x$ s binds directly or indirectly and

(b) there is an amount of energy $e>0$ such that

(b1) all direct bonds between the $x$ s have an energy greater than or equal to $e$ and

(b2') there is no extension of the $x$ s that fulfills (a) and (b1) and

(b3) $e$ is the maximal amount of energy for which (b1) is true.

\subsection{Discussion}

\subsubsection{Comparison with Van Inwagen's Concepts of Bonding}

(1) By our argument, we have provided further reasons against the criterion of contact that van Inwagen discusses and discards. Specifically, it is the argument against repulsive interactions (argument against H1) that does so. As mentioned above, solid bodies are surrounded by clouds of electrons, which repel each other. This repulsion, which is strong for small distances, gives rise to what we know as the impenetrability of a solid body and which becomes evident when two bodies are in contact. Hence, our argument against composition by repulsive interactions - repulsive interactions tend to push objects apart, 
i.e., they tend to violate the finite distance condition - also discards contact as a suitable criterion. This view is in agreement with van Inwagen's and just adds another argument.

(2) Bonding by repulsion is similar to contact in that it is also based on electromagnetic repulsion. In fact, many (but not all - see discussion point (3)) cases of bonding by repulsion occur by repulsion at contact. The two differ, however, in the following sense: Contact only requires that two objects feel each other's repulsive force and, without further qualification, tends to push objects apart; bonding by repulsion, however, requires repulsion from opposing directions such that the motion of the objects is confined and a kind of bonding occurs. This can happen through contact with one and the same object if that object has an appropriate geometry - and this explains why the geometry of parts binding by repulsion is crucial. For example, a screw and an adequate thread are designed to fit together in order to fasten parts of some object. The thread confines the motion of the screw in all directions (even the direction in which one can turn the screw might be confined due to friction). However, if the available screw is too thin or the thread is flattened from being used, there is no confinement and the fastening will not work.

A Lego house offers another interesting example of bonding by repulsion. Its bricks are constrained horizontally by their studs on top of each brick and the tubes at the bottom. Vertically, the bricks are held together by the friction between the studs and the tubes, which is also based upon molecular repulsion, causing a certain resistance against being torn apart. Since the friction is not as strong as the horizontal fastening, it is the weakest bond within the Lego house and determines its rather low robustness.

(3) Our concept of "bonding by repulsion" is in some respects similar to van Inwagen's "fastening" but differs from it essentially. Especially, fastening requires that the objects are separable in a certain way without breaking them - which is not true for bonding by repulsion. Think, for instance, of two rings that are forged in an entangled way. According to van Inwagen, these rings are not fastened, because they cannot be separated without one being destroyed. But they are clearly bound together, and the bonding occurs by repulsion. This example also shows that van Inwagen's partition of bonding into fastening, cohesion and fusion is incomplete; for the entangled rings do not fall into any of his categories (though they are clearly bound to one another).

(4) In the light of our analysis, van Inwagen's distinction between cohesion and fusion seems largely irrelevant. Both require that the objects are in contact and bound together; in the former case "there is a discernible boundary" (van Inwagen 1990, 59), while in the latter case there is not. In some cases, this distinction captures only an epistemic aspect: Compare the joining of two pieces of iron, one pair fused (resulting in a seamless junction), while the other is welded (resulting in a visible junction). In both cases the physical bonds are of the same type, so that on an ontic level there is no difference in the connec- 
tion; rather, the difference is purely epistemic: In only one case you can see that the pieces have been joined. Referring to an epistemic difference, the cohesion/fusion distinction should not play a role in metaphysical considerations.

Our analysis also suggests that even in cases where there is a physical difference, the distinction is not relevant. Consider the case that two pieces of metal are joined by a layer of glue. Involving another kind of material, the glue, this makes their connection physically different from the welding case. According to our theory, however, this does not make a qualitative difference for their status of composing a whole: What is essential is that they are bound together. If the glue binds the pieces of metal together with the same strength as the welding (admittedly, this would have to be a rather strong glue), there would not even be a quantitative difference, that is, a difference in the objects' robustness. While the physical differences between fused and glued pieces of iron might be of interest in many other contexts, if our proposal is correct, they seem to be irrelevant to the question whether these pieces of iron compose an object or not.

(5) Van Inwagen's concepts of cohesion and fusion require bonding and therefore are sufficient for composition according to our theory. But they also require contact and this makes them too narrow (they are not necessary for composition). Think about the entangled rings again: They may be in contact, but even if they are not, they are in a bound state. In a similar manner, there are also cases of bonding by attraction in which the parts are far away from one another. The earth orbiting the sun is an example of this kind: The two may never be in contact and still they are perfectly bound together. If no strong external force is applied to the rings or the celestial bodies, they can only move within a limited area and therefore fulfill the finite distance condition as well as the joint movability condition. In this sense, our theory accepts that wholes are composed without the parts touching or even coming close to each other - we call this remote bonding. Therefore, a subclass of scattered objects (i.e., objects composed of parts that are not spatio-temporally contiguous) exists, namely the subclass of (simultaneously existing) objects whose parts are remotely bound.

\subsubsection{Advantages of Bonding as a Criterion for Physical Composition}

(6) Our criterion of physical composition captures the complete ontology of solid inanimate objects as sciences like physics, chemistry or astronomy suggest it, from the smallest particles to the largest structures: On a fundamental level there are quarks and electrons as simples; three quarks at a time binding by strong nuclear interaction compose 
protons or neutrons, respectively; by the same kind of interaction, protons and neutrons compose atomic nuclei; nuclei and electrons by electromagnetic binding compose atoms. Atoms of certain kinds bind together to atomic lattices (composing salts or metals); or they bind together to form molecules; molecules can bind to form solid bodies as well; and they can also form gases, which, however, do not, in general, exist as composite objects, because molecules of gases are typically not in a bound state (exceptions are massive amounts of gas that bind gravitationally to gas planets such as Jupiter). Finally, molecules can also make up liquids, which is a special intermediate phase: Liquids consist of molecules some of which are bound to each other, but there is no all-embracing structure of bonding by attraction as can be found in solid bodies; there possibly are clusters of molecules bound together, and since these bonds change constantly, the clusters of bound molecules arise and vanish rapidly. Therefore, at best, only parts of what we conceive as a portion of liquid, say, water in a glass, exist by our criterion of composition.

Atoms and molecules in solid phases compose the natural inanimate mid-sized objects that we are used to: grains of sand, stones, pieces of wood, etc. From these objects common material artifacts are produced: tables, cars, paintings; and from mid-sized natural inanimate objects also larger natural structures are composed: planets, stars, solar systems, interstellar clouds, galaxies and, finally, the universe (the latter three, of course, only insofar as they exist in bound states). In this way we get virtually all inanimate objects that belong to a scientific ontology (and most objects that belong to folk ontology).

(7) The plausibility of bonding as a physical composition criterion partly stems from the fact that objects composed of bound parts can be moved together (joint movability condition; see Sect. 4.3). Imagine you see the upper part of a ring reaching out of a sandy beach. Since the metal-molecules are strongly bound to each other, lifting the ring's upper part will lift the whole ring. In contrast, the sand, at least if it is dry, is not bound to the ring, so it will ripple off the metal ring as soon as the latter is moved. Similarly, grabbing a coffee mug at its handle raises the whole mug. Or as the earth continuously moves around the sun, it takes with it all that is gravitationally bound to it: all the objects on its surface (including its human inhabitants) as well as its complete atmosphere. The fact that bound objects can be moved together also explains the practical relevance of demarcating objects. Often when we interact with our environment it is useful to estimate the range of our actions. What happens if I lift this part? Do I also lift other parts, or does the alleged object fall into pieces? Defining composition in terms of bonding therefore cuts nature at its causal seams.

(8) Referring to bonding energy in our criterion of composition entails the following interesting features for our theory. Firstly, it allows to graduate objects of different robustness, which is of practical relevance when we want to know how stable a certain ob- 
ject is or when we want to break it apart. Secondly, bonding energy enables us to differentiate parts of an object without going all the way down to its simples. In many cases it would be unsatisfactory to identify simples as the object's parts; rather, it often suffices to detect macroscopic parts. Consider an example: Since the bonds between the molecules composing Lego bricks are stronger than the bonding between the bricks forming a house, according to our theory (and agreeing with folk ontology), the bricks are considered as existing, macroscopic parts of the Lego house. In a similar way, the next lower level can be analyzed: The molecules making up the bricks are much more weakly bound than the atoms composing the molecules - so the molecules are genuine parts of the bricks - and so on. In other words, by referring to binding energy, our ontology contains a hierarchy of nested objects, which is typical for the scientific ontology we have been aiming at.

\subsubsection{Some Non-obvious Consequences}

(9) Which objects, according to our theory, belong to the system that we might reasonably call "planet earth"? That system includes, of course, all geological layers from the earth's core to its mantle. Clearly, it also includes objects on the earth's surface if they are fixed to it like enrooted trees. But also objects that are not fixed to the earth like animals or humans count as parts of the planet, because they are gravitationally bound to it. And for a similar reason the whole atmosphere belongs to the earth, as well as all flying objects like birds or airplanes and even satellites and the moon.

(10) Consider a billiard ball lying on a billiard table. Do the table and the ball form a whole? The ball's horizontal motions are clearly restricted by the repulsive interactions with the cushion while its vertical motion is restricted by gravitation. The latter, however, is mainly due to the earth and therefore is external to the system. However, the table has a very small gravitational force on the ball as well and for this reason the ball and the table compose an object, though with an extremely low robustness. Similarly, balls in a completely closed box compose a whole together with this box, as their motion is confined in all directions. Analogously, water in a closed bottle does compose an object with this bottle because the molecules' motion is confined by the bottle and its cap.

(11) A gecko walks on a glass surface and sticks to it by means of intermolecular bonding. According to our criterion of composition, the gecko together with the glass surface composes an object, and if the gecko walks to another object's surface, it suddenly composes a new object together with it. Similarly, a sheet of paper and a balloon com- 
pose an object if they are bound to each other because they are electrostatically charged. It runs against our intuitions to claim the existence of gecko-glass objects or balloonsheet objects. First of all, note that our claim is not that the gecko or the balloon do not exist; they do exist, according to our theory, because their parts are bound to one another. What seems counterintuitive about our claim rather is that the gecko as well as the balloon are parts of larger composite objects (with a lower robustness), namely a gecko-glass object and a balloon-sheet object. But is that really as surprising as it might seem at first sight? It is clear that the gecko and the glass or the balloon and the sheet can be moved together in such cases: If you want to bring the gecko back into its terrarium, you can lift the glass; if you want to pick up the sheet, you can pick up the balloon. And why, then, should one not accept that there is a physical body composed of the gecko and the glass? While intuitions may serve as preliminary guidelines, they should not, in our view, be regarded as a universal criterion for ontological theories. The gecko-glass and the balloonsheet might seem surprising objects, but their existence is a consequence of clear ontological principles that, as we have argued in this paper, imply a suitable ontology.

\subsubsection{Combining the Criteria: Physical Bodies and Living Organisms}

(12) What about living organisms in our picture? By what we have said, all biomolecules clearly exist. Viruses are molecules and, hence, exist as well. Cellular membranes consist of bound lipid molecules (arranged in a double layer) and exist. Inside the cell there is a rigid cytoskeleton, which exists. All molecules inside a cell's watery solution are bound by repulsion from the membrane (just like water in a closed bottle), hence cells as a whole exist. In human bodies all bones clearly exist. They are connected by strings and muscles and hence the complete musculoskeletal system exists. Also, all organs are clearly bound systems and exist. Finally, if there is any doubt, the skin packs everything together and binds all other bodily components by repulsion. In sum, according to our theory, even complex organisms like human bodies exist.

(13) Does that make van Inwagen's criterion of life redundant? For two reasons we believe it does not. First, our criterion captures organisms insofar as they are bound objects. There may, however, be organisms that consist of several parts, which are not bound in the sense required by our criterion. Depending on one's notion of organism, bee colonies, corral reefs or the like may be organisms not captured by our criterion. And if such things constitute a life, van Inwagen's criterion would cover cases of composition that the criterion for physical bonding does not. 
Second, and more importantly, our criterion does not capture the living aspect of organisms; it just captures them as physical bodies, regardless of whether they are dead or alive. It does not distinguish between a corpse and a sleeping man. In order to illustrate this difference between organisms and physical objects, recall van Inwagen's examples of two people becoming bound to one another while shaking hands (Sect. 4.2): either because their fingers become paralyzed (fastening), because they are glued together (cohesion) or because they are operated by a mad surgeon to form artificial Siamese twins (fusion). In all these cases the two bodies are clearly bound to one another: Their relative motions are confined such that moving the one will move the other. So by the criterion of bonding, a physical object is composed which has the two persons' bodies as parts. But in none of these scenarios a new organism is composed since it is not the case that the joint activities of the two bodies form a life.

(14) For these reasons we believe that an appropriate sufficient as well as necessary criterion of composition simpliciter must involve both criteria disjunctively as follows:

Criterion of composition: $\exists y$ (the $x$ s compose $y$ ) if and only if

(i) (a) every pair of the $x$ s binds directly or indirectly and

(b) there is an amount of energy $e>0$ such that (b1) all direct bonds between the $x$ s have an energy greater than or equal to $e$ and (b2) there is no extension of the $x$ s that fulfills (a) and (b1) and

(c) $e$ is he maximal amount of energy for which (b1) is true;

(ii) the activity of the $x \mathrm{~s}$ constitutes a life.

Sub-criterion (i) is sufficient for the composition of physical bodies, while subcriterion (ii) captures cases of living organisms. As we have explained, each criterion might capture cases that the other does not (there clearly are physical bodies that are not organisms; and there might be organisms that are not physical bodies); but there are also intersecting cases when both criteria claim composition. As an example for the latter case, consider a living human organism. We have said that, according to both criteria, this is a composite object. But then: Do we have two extensionally equivalent objects? So it seems: We both have a physical body as well as a living organism, and each has different criteria of identity. The former's criteria are based on bonding, while the latter's are based on living. And the problem of these two co-extensional things with different criteria of identity is analogous to the problem of the statue and the clay.

Van Inwagen (1990, 52-53, 125-127) has provided strong arguments against such coextensional objects, and we agree with him that numerically distinct co-extensional objects should be avoided. However, developing our approach in this way must be left for 
future work. Prospective investigations would also have to address questions of diachronic identity of physical bodies, which we have not touched in this paper. While in this sense our proposal is still a work in progress, is it not tempting to include all those molecules, tables and stars in one's ontology?

(15) In this way, we have expanded van Inwagen's ontology to include physical bodies. Accepting these bodies shifts van Inwagen's ontology away from nihilism: As he only accepts simples and organisms (and the cells of multicellular organisms), there are extremely small, indivisible objects and rather complex ones - but nothing in between. Physical composition by bonding, as we have developed it in this paper, yields the existence of protons, neutrons, atoms, molecules - virtually all the composite theoretical entities that a scientific realist assumes and van Inwagen denies - as well as larger structures in organisms like organs and bones, which do not appear in van Inwagen's ontology either. Thus, it bridges the gap between simples and cells as well as that between cells and multicellular organisms. Moreover, it includes the solid mid-sized objects that we are used to in everyday life like stones, cars and houses; and it also prolongs the spectrum of composed objects by larger entities like continental plates, planets, solar systems, galaxies and maybe even the universe (if it is in a bound state). Enriching the furniture of the universe in the way we have proposed here avoids closeness to nihilism, while, on the other hand, denying the existence of arbitrary undetached parts and most scattered objects lets our ontology steer clear from universalism. In this sense, it seems to us, our proposal might be a promising candidate for a via media between nihilism and universalism.

\section{References}

Aristotle. 1995. Metaphysics. In The Complete Works of Aristotle, ed. Jonathan Barnes. Princeton: Princeton University Press.

Atkins, Peter W., and Julio de Paula. 2011. Physical Chemistry for the Life Sciences. 2nd ed. Oxford: Oxford University Press.

Calosi, Claudio, and Gino Tarozzi. 2014. Parthood and composition in quantum mechanics. In Mereology and the sciences: Parts and wholes in the contemporary scientific context, ed. Claudio Calosi and Pierluigi Graziani, 53-84. Cham: Springer.

Carmichael, Chad. 2015. Toward a commonsense answer to the Special Composition Question. Australasian Journal of Philosophy 93: 475-490.

Chant, Sara R. 2006. The Special Composition Question in action. Pacific Philosophical Quarterly 87: 422-441.

Christen, Hans R., and Gerd Meyer. 1997. Grundlagen der allgemeinen und anorgani- 
schen Chemie. Frankfurt a. M.: Salle \& Sauerländer.

Evans, Gareth. 1978. Can there be vague objects? Analysis 38: 208.

Hoffman, Joshua, and Gary S. Rosenkrantz. 1997. Substance: Ist nature and existence. London: Routledge

Hübner, Johannes. 2007. Komplexe Substanzen. Berlin: de Gruyter.

Lewis, David K. 1983. New work for a theory of universals. Australasian Journal of Philosophy, 61: 343-377.

—. 1991. Parts of classes. Oxford: Basil Blackwell.

Locke, John. [1690] 1975. In An essay concerning human understanding, ed. Peter H. Nidditch. Oxford: Oxford University Press.

Mancin, Giacomo. 2012. On the problem of vague existence. Doctoral thesis, Università Ca'Foscari, Venice, http://dspace.unive.it/handle/10579/1191. Accessed 3 Sept 2015.

Maudlin, Tim. 1998. Part and whole in quantum mechanics. In Interpreting bodies: Classical and quantum objects in modern physics, ed. Elena Castellani, 46-60. Princeton: Princeton University Press.

Povh, Bogdan, Klaus Rith, Christoph Scholz, Frank Zetsche and Werner Rodejohann. 2015. Particles and Nuclei. Trans. Martin Lavelle. 7th ed. Heidelberg: Springer. (Translation of the 9th German ed., Teilchen und Kerne, 2014; 1st German ed. 1993)

Schaffer, Jonathan. 2010. Monism: The priority of the whole. Philosophical Review, 119 : 31-76.

Sider, Theodor. 2013. Against parthood. In Oxford studies in metaphysics: Volume 6, ed. Karen Bennett and Dean W. Zimmerman, 237-293. Oxford: Oxford University Press.

Silva Jr., Paul. 2013. Ordinary objects and series-style answers to the Special Composition Question. Pacific Philosophical Quarterly, 94: 69-88.

Tallant, Jonathan. 2014. Against mereological nihilism. Synthese 191: 1511-1527.

Thomasson, Amie L. 2007. Ordinary objects. Oxford: Oxford University Press.

van Inwagen, Peter. 1981. The doctrine of arbitrary undetached parts. Pacific Philosophical Quarterly 62: 123-137.

—. 1987. When are objects parts? Philosophical Perspectives 1: 21-47.

—. 1990. Material beings. Ithaca: Cornell University Press. 